\title{
Formulation And Evaluation Of Repaglinide Biphasic Mini Tablets
}

\author{
P. Sandhya ${ }^{1}$, Sameera Khan ${ }^{2}$ \\ Department Of Pharmaceutics, Shadan Women's College Of Pharmacy, Khairtabad.
}

\begin{abstract}
Repaglinide is an anti-diabetic drug used extensively in the treatment of diabetes type II. The present study was carried out to formulate and evaluate biphasic floating mini tablets of Repaglinide. These mini tablets were encapsulated in a capsule. Immediate release mini tablet (IRMT) were manufactured by direct compression using various superdisintegrating agents, each mini tablet containing $2 \mathrm{mg}$ Repaglinide. Sustained release mini tablet (SRMT) were formulated using various polymers, each mini tablet containing $4 \mathrm{mg}$ Repaglinide. The prepared mini tablets were subjected to pre and post compressional parameters and the values were within the prescribed limits. The drug-excipient compatibility studies were performed using FTIR techniques. The invitro performance showed the desired biphasic behaviour. Capsules were filled with individual mini tablets to deliver 6mg of Repaglinide designed to provide a multi particulate delivery of drug as immediate and sustained dissolution release profiles.
\end{abstract}

Keywords: mini tablets, Repaglinide, encapsulated biphasic mini tablets, floating drug delivery system,floating lag time.

\section{Introduction}

Oral controlled release drug delivery systems can be classified in two broad groups

1. Single unit dosage forms (SUDFs), such as tablets or capsules, and

2. Multiple unit dosage forms (MUDFs), such as granules, pellets or minitablets.

Single unit: Single unit dosage forms include matrix tablet or coated/uncoated tablet or capsules and consist of only one unit, e.g. osmotic tablet.

Multiple-unit: The basic concept of multiple-unit systems is that the dose of the active ingredient is released by the individual subunits (like, pellets, minitablets), and the functionality of the entire dose depends on the quality of the subunits ${ }^{[1]}$.

Advantages of multiple unit dosage forms

1. Local irritation and side effects are reduced and more consistent plasma levels are achieved ${ }^{(2)}$.

2. Coated MUDFs prevents the risk of dose dumping due to the use of multiple particles.

3. The combination of different multiple particles may reduce the daily required number of drugs, which can improve patient compliance.

4. MUDFs may seem costlier than SUDFs in the short term; but causes significant savings, lower treatment failure rate, lower case-fatality ratios, reduction in development of resistance, more predictable gastric emptying and consequently less money needed for the development of new products in long term therapy ${ }^{[3]}$.

Disadvantages of multiple unit dosage forms

1. The main disadvantage of MUDFs is the complex production process.

2. MUDFs can be bigger in volume than tablets which can effect patient compliance.

Minitablets ${ }^{[4]}$

Mini tablets are flat or curved tablets with a 1.0-3.0 mm diameter which, for pharmaceutical use, are normally filled in hard gelatine capsules

Mini tablets can be an alternative to normally sized tablets, if there are specific preparation requirements as regards the following aspects:

- Release profile/ drug absorption

- Constituents

- A comparison of pellets/ mini tablets

\section{Encapsulated biphasic minitablets}

Encapsulated biphasic mini tablet system is aimed to reduce the size of the tablet such that it can be enclosed in a capsule, and then deploy tablets with different release properties within the one encapsulated mini tablet. This biphasic system comprises of immediate release mini tablets (IRMT) and sustained release mini tablets (SRMT) 
in a hard gelatine capsule. These mini tablets can also be filled in HPMC capsule. The main objective of this study is to achieve desired target product profile (DPP) for a period of 12 hours.

Biphasic delivery systems are designed to release a drug at two different rates or in two different periods of time: they are either quick/slow or slow/quick. A quick/slow release system provides an initial burst of drug release followed by a constant rate (ideally) of release over a defined period of time and in slow/quick release system provides release vice versa ${ }^{[7]}$.

Several approaches have been proposed to retain the dosage forms in the stomach. These methods include bioadhesive system, swelling system and expanding system and floating system.

Gastroretentive drug delivery systems (GRDDS) can improve the controlled delivery of drugs that have an absorption window by continuously releasing the drug for a prolonged period of time before it reaches its absorption site thus ensuring its optimal bioavailability ${ }^{[8,9]}$.

Floating mini tablets - Floating drug delivery system (FDDS) possesses a bulk density lower than gastric fluids and thus remain buoyant in the gastric fluids for a prolonged period without affecting the gastric emptying rate. While the system is floating on the gastric content, the drug released slowly at desired rate from the system $^{[10]}$.Based on the mechanism of buoyancy, two distinctly different technologies, non-effervescent and effervescent systems have been utilized in the development of FDDS. In effervescent systems a gas generating agent usually sodium bicarbonate or sodium carbonate is mixed with matrices prepared with swellable polymers, when the systems comes in contact with gastric fluids, the carbondioxide is liberated by the acidity of gastric contents and the gas is entrapped in the viscous hydrocolloid. Thus produces an upward motion of the system maintaining buoyancy ${ }^{[11]}$.

\section{Repaglinide}

It is the first member of the class of oral hypoglycaemics designed to normalize the meal time glucose excursions. It is administered before each major meal to control postprandial hyperglycemia; the dose may be omitted if a meal is missed. Because of short lasting action it may have a lower risk of serious hypoglycemia. Side effects are mild headache, dyspepsia, arthralgia, and weight gain. After oral administration, Repaglinide is rapidly and completely absorbed from the gastrointestinal tract. Repaglinide is a white to off-white powder with molecular formula $\mathrm{C}_{27} \mathrm{H}_{36} \mathrm{~N}_{2} \mathrm{O}_{4}$ and a molecular weight of 452.6 , poorly soluble in water. Its having half-life (0.9-1 hour) and usually administered with a starting dose of $0.5 \mathrm{mg}$ before each meal as immediate release tablets . The maximum tolerable dose of Repaglinide is $16 \mathrm{mg}$ per day. The blood level fluctuation resulting from this frequent administration leads to extrpyramidal symptoms.

\section{Literature review}

D. Karthikeyan et al., ${ }^{[1]}$ Investigated the feasibility of formulating a biphasic delivery system using mini-tablets in hard gelatin capsules delivering drug with a variety of release profiles. Aceclofenac (NSAID analgesic) were chosen as model drug. Immediate release minitablets (IRMT) were manufactured by direct compression using microcrystalline cellulose and cross carmellose as a superdisintegrant, each mini-tablet containing approximately $25 \mathrm{mg}$ Aceclofenac (AFC). Sustained release mini-tablets (SRMT) were formulated using various ratios of HPMC K4M and HPMC K100M. Capsules were filled with individual mini-tablets to deliver $100 \mathrm{mg}$ of AFC. The IR and SRMT were combined in the capsule designed to provide a pulsatile or multi-phase delivery of drug as immediate and sustained dissolution release profiles. Capsules containing AFC immediate release mini-tabs were shown to release nearly $100 \%$ of the drug within 60 minutes. Capsules containing SRMT released $98 \%$ of AFC over 12 hours. The feasibility of delivering a drug in biphasic immediate sustained release manner was reputable by combining mini-tablets in a hard gelatin capsule.

Noorana Tehseen et al., ${ }^{[2]}$ worked on design and characterization of twice daily mini-tablets formulation of pregabalin in order to improve the half life and bioavailability. The system comprises of 15 matrix mini-tablets weighing $25 \mathrm{mg}$ encapsulated in HPMC capsule (size1). For achieving the sustain release profile, various viscosity grades of Hydroxy propyl methyl cellulose polymer (HPMC K4M, K15M, K100M) were used. The mini-tablets were prepared by direct-compression method. The prepared mini-tablets were subjected for pre-compressional and post-compressional parameters. The compatibility of drug with other ingredients was checked by FTIR studies. Stability study carried out as per ICH guidelines for three months.

Carla M. Lopes et al., ${ }^{[3]}$ developed compressed mini tablets as a biphasic delivery system designed for zero-order sustained drug release. The outer layer that fills the void spaces between the mini-tablets was formulated to release the drug in a very short time (fast release), while the mini-tablets provided a prolonged release. Different composition (HPMC or EC) and number (10 or 21) of mini-tablets were used to obtain different drug release rates. The in vitro performance of these systems showed the desired biphasic behaviour: the drug contained in the fast releasing phase (powder enrobing the mini-tablets) dissolved within the first 2 $\mathrm{min}$, whereas the drug contained in the mini-tablets was released at different rates, depending up on formulation. 
Based on the release kinetic parameters calculated, it can be concluded that mini-tablets containing HPMC were particularly suitable approaching to zero-order (constant) release over $8 \mathrm{~h}$ time periods.

Jitender Joshi et al., ${ }^{[4]}$ developed a once-daily sustained release matrix tablet of Repaglinide using sodium alginate as release controlling factor. In order to achieve required sustained release profile tablets were compressed using sodium CMC, sodium alginate, Magnesium Stearate, and PVP. Six different formulation of Repaglinide were prepared by using different ratio of drug: polymer. The tablet was characterized by hardness, wetting time, weight variation and In Vitro Drug Release which shows the satisfactory result. All batches of solid matrix tablets were satisfactory in terms of dissolution profile. The batches of all formulations, MT5 batch [Sodium CMC With drug (1:3)] showed more release than the other concentration and better results. The MT5 batch of Solid matrix tablets was found to be $96.0 \%$ drug release in 300 minutes.

\section{Objectives of the study}

The objective of this work was the formulation and invitro evaluation of biphasic mini tablets of Repaglinide.

\section{Materials \& Methodology}

Materials : Repaglinide,magnesium stearate, talc, lactose, cross povidone, sodium starch glycolate, cross carmellose sodium, micro crystalline cellulose, HPMC K4M, HPMC K15M, HPMC K100M, ethyl cellulose, sodium bicarbonate.

\section{Preformulation study}

\section{Scanning of Repaglinide in $0.1 \mathrm{~N} \mathrm{HCl}$}

The solution containing $100 \mu \mathrm{g} / \mathrm{ml}$ of Repaglinide in $0.1 \mathrm{~N} \mathrm{HCl}$ was prepared and scanned over the wavelength of $200-400 \mathrm{~nm}$ against $0.1 \mathrm{~N} \mathrm{HCl}$ as blank using double beam UV spectrophotometer. The plot of absorbance vs wavelength was recorded using double beam UV spectrometer.

\section{Drug - Excipients interaction study}

FTIR Spectroscopy: The study was performed by taking $1 \mathrm{mg}$ sample in $200 \mathrm{mg} \mathrm{KBr}$. The mixture was taken in a diffuse reflectance sampler and the spectrum was recorded by scanning in the wavelength region of using FTIR instrument.

Formulation of immediate release mini tablets: Tablets were prepared by direct compression method.

Table1: Composition of immediate release Repaglinide mini tablets IF1-IF6 (Direct Compression)

\begin{tabular}{|l|r|r|r|r|r|r|}
\hline Ingredients & IF1 & IF2 & IF3 & IF4 & IF5 & IF6 \\
\hline Drug & 2 & 2 & 2 & 2 & 2 & 2 \\
\hline SSG & 10 & 20 & - & - & - & - \\
\hline CP & - & - & 10 & 20 & - & - \\
\hline CCS & - & - & - & - & 10 & 20 \\
\hline Mag.stearate & 2 & 2 & 2 & 2 & 2 & 2 \\
\hline Talc & 2 & 2 & 2 & 2 & 2 & 2 \\
\hline Lactose & 37 & 12 & 37 & 12 & 37 & 12 \\
\hline & & 50 & 50 & 50 & 50 & 50 \\
Total wt. & 50 & 50 & & & \\
\hline
\end{tabular}

Table 2: Composition of sustained release Repaglinide mini tablets SF1 - SF6 (Direct Compression)

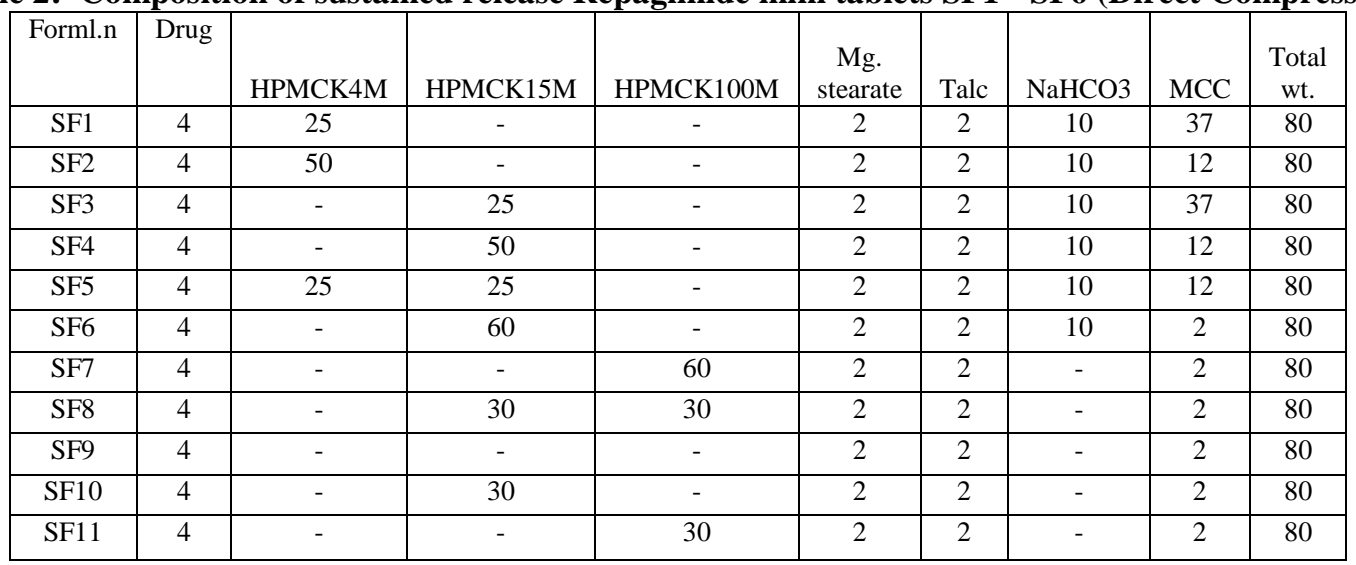


SSG - sodium starch glycolate; CP- cross povidone; CCS- cros carmellose sodium; mag,st- magnesium stearate

Evaluation of biphasic mini tablets of Repaglinide

Precompression parameters

The granules were evaluated for bulk density, tapped density, Carr's index, Hausner's ratio and angle of repose.

Post compression parameters: The prepared sustained release and immediate release mini tablets were evaluated for thickness, hardness, weight variation test, invitro release study and determination of buoyancy lag time ,total floating time and swelling index .

\section{Preformulation Study}

\section{Results \& Discussion}

UV spectrum of Repaglinide in $0.1 \mathrm{~N} \mathrm{HCl}$

UV spectrum of Repaglinide in $0.1 \mathrm{~N} \mathrm{HCl(fig} \mathrm{6)} \mathrm{shows} \mathrm{that} \mathrm{the} \mathrm{drug} \mathrm{had} \mathrm{a} \lambda_{\max }$ of $242 \mathrm{~nm}$ Drug-excipients interaction study

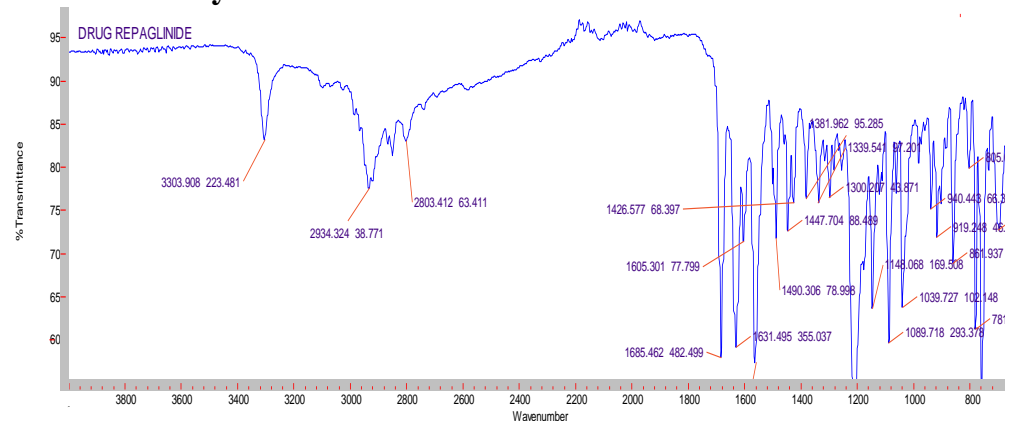

Figure 1: FTIR spectra of Repaglinide

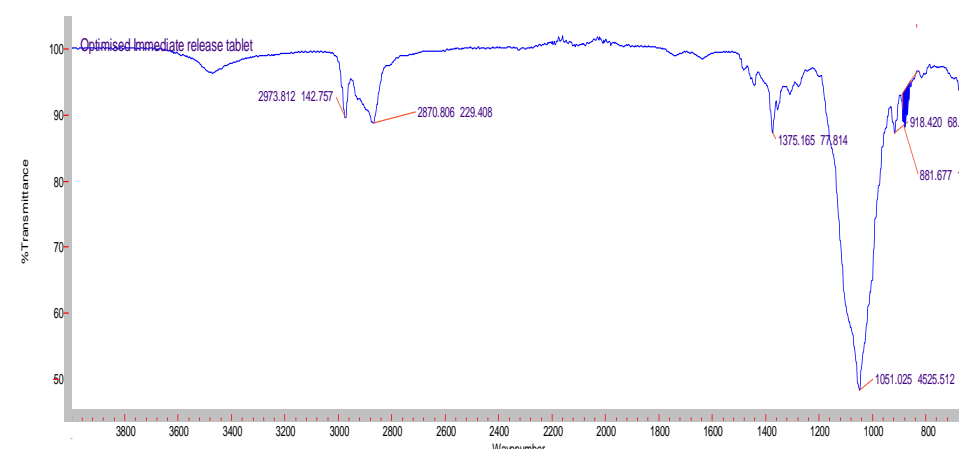

Figure 2: FTIR spectra of optimised immediate release tablet of Repaglinide

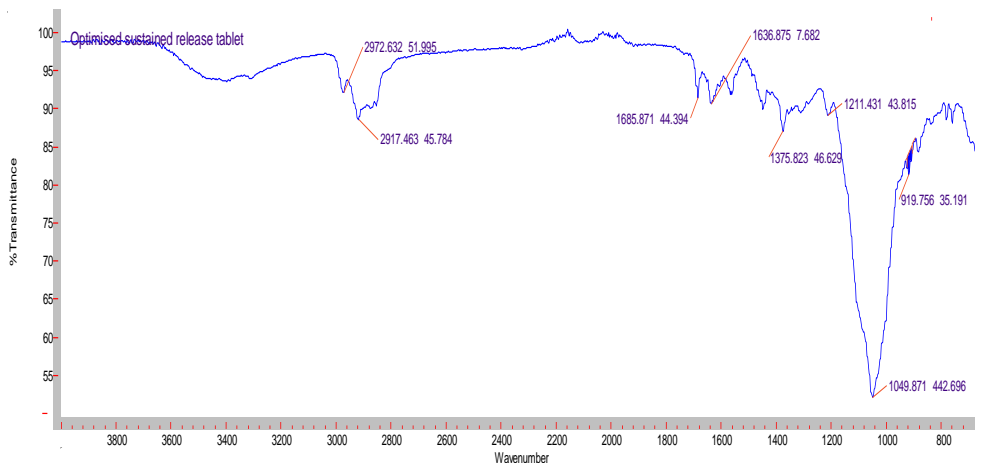


Figure 3: FTIR spectra of optimised sustained release tablet of Repaglinide

Table 3: FTIR data interpretation

\begin{tabular}{|c|l|l|l|l|}
\hline s. no & \multicolumn{1}{|c|}{ Formulations } & $\begin{array}{c}\text { Wave number in } \\
\text { formulation }\left(\mathrm{cm}^{-1}\right)\end{array}$ & $\begin{array}{c}\text { Characteristic wave } \\
\text { number range }\left(\mathrm{cm}^{-1}\right)\end{array}$ & $\begin{array}{c}\text { Bond nature and bond } \\
\text { attributed }\end{array}$ \\
\hline 1 & Pure drug & 3303.90 & $3200-3400$ & NH stretching \\
\hline 2 & Optimized IRMT & 2973.81 & $2800-3000$ & $\begin{array}{l}\text { C-H aliphatic stretching } \\
\text { of aldehyde }\end{array}$ \\
\hline 3 & Optimized SRMT & 2917.48 & $2800-3000$ & C-H aromatic stretching \\
\hline
\end{tabular}

\section{Evaluation of tablets}

Post compression parameters:

The evaluation parameters like thickness, hardness, friability and weight variation of immediate and sustained release formulations are indicated in Table 4, 5.

Table 4: Evaluation of thickness, hardness, friability and weight variation if immediate release mini tablet (IF1- IF6)

\begin{tabular}{|c|l|l|l|l|}
\hline Formulation & Thickness $^{\mathrm{A}}(\mathrm{mm})$ & $\begin{array}{l}\text { Hardness }^{\mathrm{B}} \\
\left(\mathrm{kg} / \mathrm{cm}^{2}\right)\end{array}$ & Friability (\%) & $\begin{array}{l}\text { Weightvariation(mg } \\
\mathrm{n}=3\end{array}$ \\
\hline IF1 & $2.26 \pm 0.025$ & $3.0 \pm 0.07$ & 0.38 & $50 \pm 0.46$ \\
\hline IF2 & $2.21 \pm 0.016$ & $3.0 \pm 0.11$ & 0.37 & $50 \pm 0.24$ \\
\hline IF3 & $2.20 \pm 0.026$ & $3.0 \pm 0.23$ & 0.39 & $49 \pm 1.13$ \\
\hline IF4 & $2.25 \pm 0.031$ & $3.2 \pm 0.14$ & 0.43 & $49 \pm 1.21$ \\
\hline IF5 & $2.24 \pm 0.010$ & $3.1 \pm 0.22$ & 0.47 & $50 \pm 0.76$ \\
\hline IF6 & $2.30 \pm 0.030$ & $3.0 \pm 0.23$ & 0.48 & $50 \pm 0.93$ \\
\hline
\end{tabular}

$\mathrm{A}=$ Average of 3 readings \pm S.D $\quad \mathrm{B}=$ Average of 3 readings \pm S.D

Table 5: Evaluation of sustained release mini tablet SF1- SF11

\begin{tabular}{|l|l|l|l|l|}
\hline Formulations & Thickness $(\mathbf{m m})$ & Hardness $\left(\mathbf{k g} / \mathbf{c m}^{2}\right)$ & Friability $(\boldsymbol{\%})$ & $\begin{array}{l}\text { Weightvariation } \\
(\mathbf{m g}) \mathbf{n}=\mathbf{3}\end{array}$ \\
\hline SF1 & $2.51 \pm 0.0381$ & $3.1 \pm 0.10$ & 0.45 & $80 \pm 0.35$ \\
\hline SF2 & $2.59 \pm 0.025$ & $3.4 \pm 0.173$ & 0.51 & $80 \pm 0.46$ \\
\hline SF3 & $2.47 \pm 0.0361$ & $3.5 \pm 0.308$ & 0.38 & $79 \pm 0.29$ \\
\hline SF4 & $2.52 \pm 0.0308$ & $3.3 \pm 0.316$ & 0.42 & $80 \pm 0.41$ \\
\hline SF5 & $2.57 \pm 0.030$ & $3.0 \pm 0.141$ & 0.35 & $78 \pm 1.21$ \\
\hline SF6 & $2.42 \pm 0.055$ & $3.4 \pm 0.308$ & 0.49 & $79 \pm 0.78$ \\
\hline SF7 & $2.54 \pm 0.0387$ & $3.1 \pm 0.201$ & 0.32 & $80 \pm 0.32$ \\
\hline SF8 & $2.57 \pm 0.0353$ & $3.0 \pm 0.223$ & 0.37 & $80 \pm 0.16$ \\
\hline SF9 & $2.54 \pm 0.0651$ & $3.5 \pm 0.30$ & 0.39 & $80 \pm 0.33$ \\
\hline SF10 & $2.47 \pm 0.0380$ & $3.3 \pm 0.212$ & 0.44 & $80 \pm 0.27$ \\
\hline SF11 & $2.41 \pm 0.0254$ & $3.1 \pm 0.173$ & 0.33 & $80 \pm 0.11$ \\
\hline
\end{tabular}
$\mathrm{A}=$ Average of 3 readings \pm S.D
$\mathrm{B}=$ Average of 3 readings \pm S.D

Table 6: Evaluation of disintegration time of immediate release mini tablets (IF1 - IF6)

\begin{tabular}{|r|l|}
\hline $\begin{array}{l}\text { Immediate } \\
\text { release } \\
\text { formulations }\end{array}$ & Disintegration time (mins) \\
\hline IF1 & 7 \\
\hline IF2 & 6 \\
\hline IF3 & 9 \\
\hline IF4 & 8 \\
\hline IF5 & 12 \\
\hline IF6 & 13 \\
\hline
\end{tabular}


Table 7: Dissolution data of immediate release formulations IF1 - IF6

\begin{tabular}{|r|l|l|l|l|l|l|}
\hline Time(min) & IF1 (\%) & IF2 (\%) & IF3 (\%) & IF4 (\%) & IF5 (\%) & IF6 (\%) \\
\hline 0 & 0 & 0 & 0 & 0 & 0 & 0 \\
\hline 1 & 55.4 & 52.88 & 49.03 & 42.9 & 39.42 & 40.38 \\
\hline 3 & 64.8 & 64.9 & 54.8 & 66.3 & 57.4 & 49.86 \\
\hline 5 & 77.4 & 88.55 & 67.7 & 77.9 & 62.5 & 51.98 \\
\hline 10 & 91.75 & 94.7 & 81.25 & 85.43 & 69.75 & 68.2 \\
\hline 15 & 97.1 & 99.7 & 92.65 & 95.32 & 95.32 & 70 \\
\hline 30 & 108.65 & - & 109.13 & 102.4 & 99.8 & 76.92 \\
\hline 45 & - & - & - & - & - & 87.1 \\
\hline 60 & - & - & - & - & - & 99.7 \\
\hline
\end{tabular}

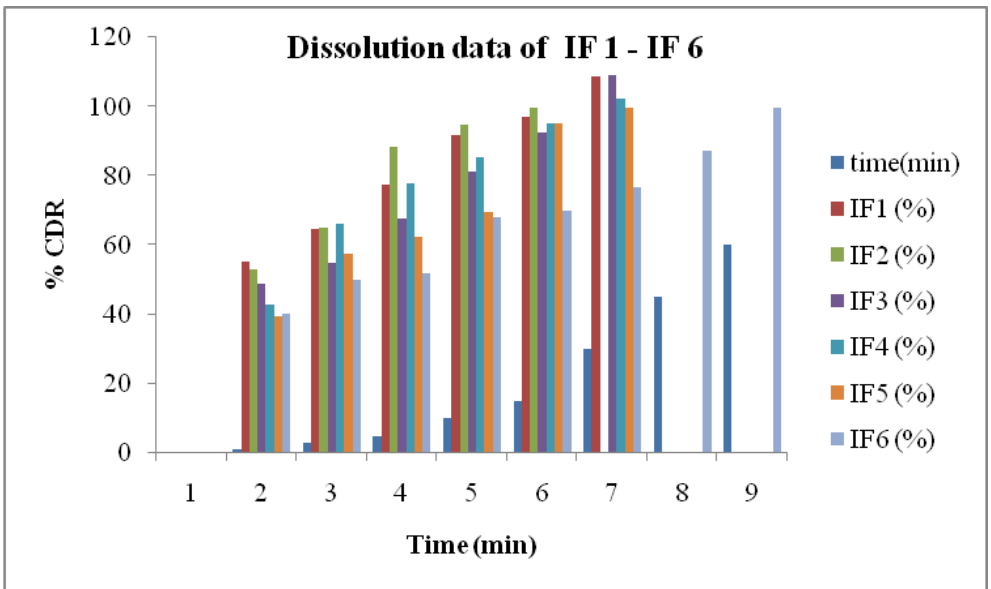

Figure 4: Dissolution data of immediate release formulations IF1 - IF6

Table 8: Evaluation of floating lag time (FLT) and total floating time (TFT)of floating sustained release mini tablets (SF1-SF11)

\begin{tabular}{|l|l|l|}
\hline Formulation & \multicolumn{1}{|c|}{ Floating Lag Time (sec) } & \multicolumn{1}{|c|}{ Total Floating Time (hrs) } \\
\hline SF1 & $88 \pm 1.88$ & Upto 24 hrs \\
\hline SF2 & $72.56 \pm 1.67$ & Upto 24 hrs \\
\hline SF3 & $80 \pm 1.98$ & Upto 24 hrs \\
\hline SF4 & $65.57 \pm 2.78$ & Upto 24 hrs \\
\hline SF5 & $55.5 \pm 2.44$ & Upto 24 hrs \\
\hline SF6 & $47.66 \pm 1.90$ & Upto 24 hrs \\
\hline SF7 & $45 \pm 2.10$ & Upto 24 hrs \\
\hline SF8 & $37 \pm 2.33$ & Upto 24 hrs \\
\hline SF9 & $32 \pm 1.97$ & Upto 24 hrs \\
\hline SF10 & $31 \pm 2.98$ & Upto 24 hrs \\
\hline SF11 & $28 \pm 1.77$ & Upto 24 hrs \\
\hline
\end{tabular}

Table 9: Dissolution data of sustained release formulations SF1 - SF 11

\begin{tabular}{|c|c|c|c|c|c|c|c|c|c|}
\hline Forml.n & & $0.5 \mathrm{hrs}$ & $1 \mathrm{hrs}$ & $2 \mathrm{hrs}$ & $3 \mathrm{hrs}$ & $4 \mathrm{hrs}$ & $6 \mathrm{hrs}$ & $8 \mathrm{hrs}$ & $10 \mathrm{hrs}$ \\
& $0 \mathrm{hrs}$ & & & & & & & \\
\hline SF1 & 0 & 44.5 & 69.7 & 88 & 97.3 & 102.16 & - & - \\
\hline SF2 & 0 & 37.6 & 49.8 & 64.9 & 89.3 & 99.9 & - & - \\
\hline SF3 & 0 & 42.6 & 59.2 & 63.9 & 72.8 & 95.19 & - & - & - \\
\hline SF4 & 0 & 32.3 & 68.5 & 79.2 & 90.86 & 95.19 & - & - & - \\
\hline SF5 & 0 & 28.8 & 44.6 & 69.2 & 85.81 & 98.79 & - & - & - \\
\hline SF6 & 0 & 32.45 & 43.99 & 53.36 & 64.75 & 72.83 & 83.65 & 97.3 & - \\
\hline SF7 & 0 & 31.55 & 55.52 & 77.8 & 78.6 & 88.7 & 96.72 & 106.7 & - \\
\hline SF8 & 0 & 41.82 & 54.08 & 66.24 & 72.1 & 75.72 & 86.77 & 95.19 & - \\
\hline SF9 & 0 & 38.79 & 49.75 & 58.41 & 69.35 & 72.58 & 88.9 & 96.3 & - \\
\hline SF10 & 0 & 29.65 & 49.75 & 51.88 & 69.36 & 78.45 & 89.98 & 95.3 & 102.4 \\
\hline SF11 & 0 & 20.19 & 38.22 & 54.56 & 61.29 & 70.67 & 86.53 & 90.14 & 96.23 \\
\hline
\end{tabular}




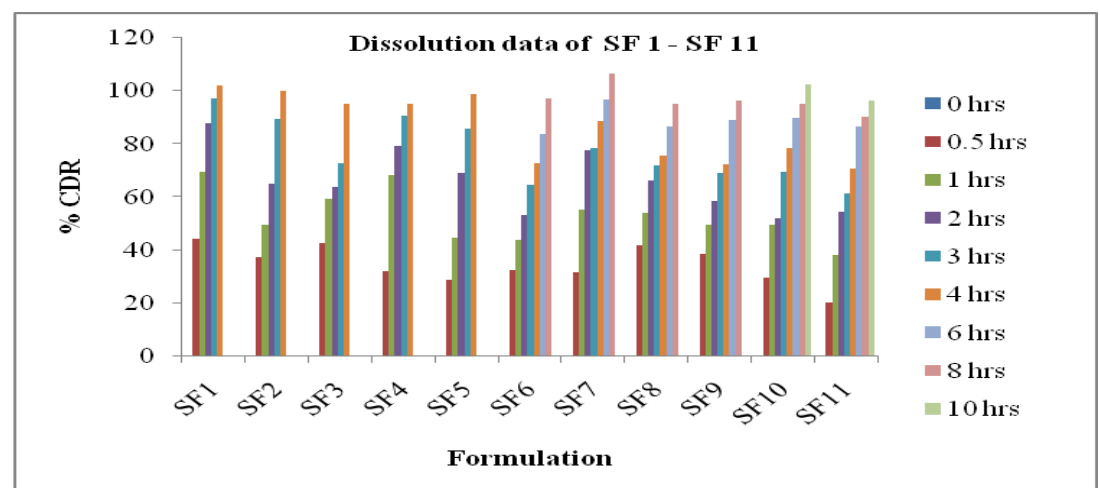

Figure 5: Dissolution data of sustained release formulations SF1 - SF 11

The percentage drug released in the first 30 min was similar in the all formulations. However, in IRMT IF2, $99.7 \%$ of the Repaglinide was released within the first 15 min Fig. 4 . In-vitro release tests were carried out for SRMT , Fig. 5 the optimized formulation SRMT SF11 released 90\% of the Repaglinide within 8 hrs and $96.23 \%$ within $10 \mathrm{hrs}$.

\section{Drug release Kinetics}

Table10: Model fitting for formulation optimised sustained release formulation SF11

\begin{tabular}{|c|c|c|c|c|c|c|}
\hline$\% \mathrm{CDR}(\mathrm{Q})$ & time(T) & $\operatorname{root}(\mathrm{T})$ & $\log (\%)$ Release & LOGT $(\%)$ & $\log (\%)$ remain & $\begin{array}{l}\text { Release } \\
\text { rate }\end{array}$ \\
\hline 0 & 0 & 0 & & & 2 & \\
\hline 20.19 & 0.5 & 0.707 & 1.305 & -0.301 & 1.902 & 40.38 \\
\hline 38.22 & 1 & 1 & 1.582 & 0 & 1.791 & 38.22 \\
\hline 54.56 & 2 & 1.414 & 1.737 & 0.301 & 1.657 & 27.28 \\
\hline 61.29 & 3 & 1.732 & 1.787 & 0.477 & 1.588 & 20.43 \\
\hline 70.67 & 4 & 2 & 1.849 & 0.602 & 1.467 & 17.668 \\
\hline 86.53 & 6 & 2.449 & 1.937 & 0.778 & 1.129 & 14.422 \\
\hline 90.14 & 8 & 2.828 & 1.955 & 0.903 & 0.994 & 11.268 \\
\hline 96.23 & 10 & 3.162 & 1.983 & 1 & 0.576 & 9.623 \\
\hline
\end{tabular}

\begin{tabular}{|l|l|l|l|l|l|}
\hline 1/cum. \% release & $\begin{array}{l}\text { Peppas log } \\
\text { Q/100 }\end{array}$ & $\begin{array}{l}\text { \% drug } \\
\text { remaining }\end{array}$ & Q01/3 & Qt1/3 & Q01/3-Qt1/3 \\
\hline & & 100 & 4.642 & 4.642 & 0 \\
\hline 0.0495 & -0.695 & 79.81 & 4.642 & 4.305 & 0.336 \\
\hline 0.0262 & -0.418 & 61.78 & 4.642 & 3.953 & 0.688 \\
\hline 0.0183 & -0.263 & 45.44 & 4.642 & 3.568 & 1.073 \\
\hline 0.0163 & -0.213 & 38.71 & 4.642 & 3.383 & 1.259 \\
\hline 0.0142 & -0.151 & 29.33 & 4.642 & 3.084 & 1.558 \\
\hline 0.0116 & -0.063 & 13.47 & 4.642 & 2.379 & 2.262 \\
\hline 0.0111 & -0.045 & 9.86 & 4.642 & 2.144 & 2.497 \\
\hline 0.0104 & -0.017 & 3.77 & & 1.556 & \\
\hline
\end{tabular}
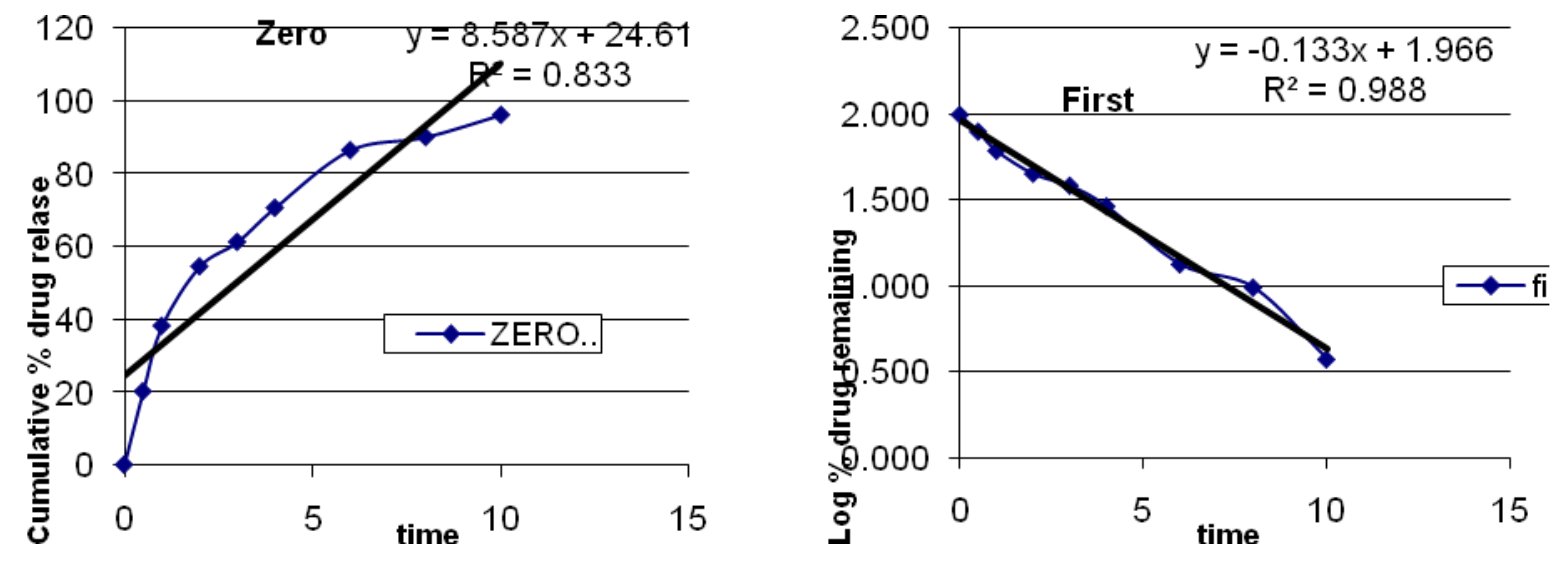

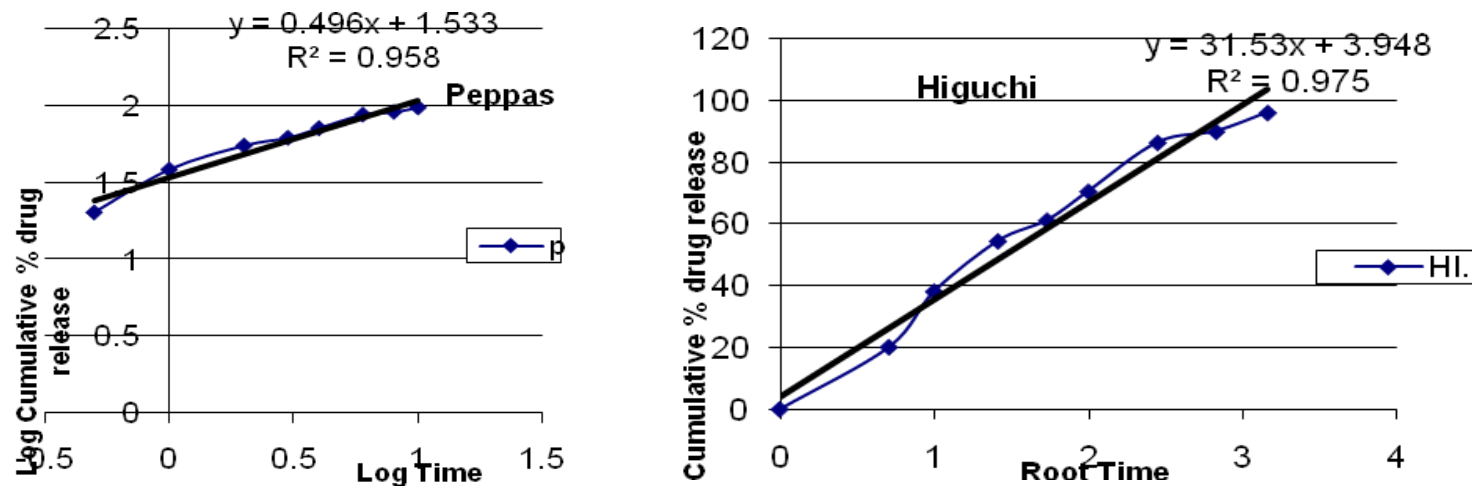

Figure 6: Model fitting for formulation optimised sustained release formulation SF11

The mechanism of drug release for the optimized mini tablet formulation was based on regression coefficient (r2) value. From the Table 10 it can be concluded that the drug release followed first order model Fig. 6.

\section{Conclusion}

In the present study, an attempt was made to develop a biphasic mini tablets in a capsule system containing immediate and floating sustained release mini tablet. Because of their physical characteristics, minitablets tend to keep their integrity after compression, making more difficult the fracturing process of these subunits.

IF2 was optimised in (IRMT) immediate release minitablet containing SSG as a super disintegrating agent and SF11 was optimised in (SRMT) sustained release minitablet containing HPMC K100M and Ethyl cellulose along with other common tablet excipients to control as well as extend the drug release over a period of $10 \mathrm{hrs}$. The optimised mini tablets placed in a capsule showed satisfactory results in several in vitro tests. Number of mini tablets can be filled depending upon the size of the capsule shell and the diameter or weight of mini-tablets.

\section{References}

[1] D. Karthikeyan, A.Vijayalaxmi, C. Santhosh Kumar, Formulation and evaluation of biphasic Delivery system of aceclofenac minitablets in Hard gelatin capsules, ISSN: 2277 - 2782, international journal of novel trends in pharmaceutical sciences.

[2] Noorana Tehseen, Vinay Rao, Mohd Abdul Hadi, design and characterization of twice daily mini-tablets formulation of pregabalin, International journal of pharmacy and pharmaceutical sciences, ISSN-0975-1491, VOL 5, Suppl 1, 2013

[3] Carla M. Lopes, Jos'e Manuel Sousa Lobo, Jo ao F. Pinto, Paulo Costa, Compressed mini-tablets as a biphasic delivery system, International Journal of Pharmaceutics 323 (2006) 93-100

[4] Jitender Joshi, Lata Bhakuni and Sachin Kumar, Formulation and evaluation of solid matrix tablets of Repaglinide, Der Pharmacia Sinica, 2012, 3 (5):598-603, Pelagia Research Library, ISSN: 0976-8688

[5] Gross, S.T., Hoffman, A., Donbrow, M., Benita, S., 1986. Fundamentals of the release mechanism interpretation in multiparticulate systems: the prediction of the commonly observed release equations from statistical population models for particle ensembles. Int. J. Pharm. 29, 213-222.

[6] Bechgaard and Nielsen, 1978; Davis et al .,1986; Voigt, 2006b

[7] Dashora K, Saraf S and Saraf S Development of sustained release multicomponent microparticulate system of Diclofenac sodium and Tizanidine hydrochloride. Int J Pharm Sci and nanotechnology. 2008;1: 98-105.

[8] http://www.ritter-pharma-technik.de/english/minitablettierung.html

[9] Thomson S.A., Tuleu C., Wong I.C.K, Keady S., Pitt K.G., Sutcliffe A.G., Minitablets: New modality to deliver medicines to preschool-aged children, Pediatrics 2009;123e235-e238.

[10] Mako to Ishida, Kenichi Abe, Munoru Hashezime, and Masco Kawamura. A novel approach to sustained pseudoephedrine release: Differentially coated Mini-tablets in HPMC capsules. Int J Pharm 2008; 46-52.

[11] Huang J, Kao H, Wu XY. The pH-dependent biphasic release of Azidothymidine from a layered composite of PVA disks and P (MMA/MAA) spheres. Toronto, Ontario, Canada M5S2S2, 1999

[12] Chawla, G., Gupta, P., Koradia, V. and Bansal, A. K., Gastroretention- A means to address regional variability in intestinal drug absorption, Pharm.Tech., July 2003, 27(7): 50-51.

[13] Vyas, S.P. \& Khar., “ Targeted and Controlled Drug Delivery Novel Carrier System”, Ist Ed., CBS Publishers and Distributors, New Delhi, 2002, pp. 417-54.

[14] Gopalakrishnan S, Chenthilnathan A. Floating drug delivery systems: A Review. Journal of Pharmaceutical Science andTechnology. 3 (2); 2011: 548-554.

[15] Formulation and Characterization of Metformin Hydrochloride Floating Tablets, Ashok A. Hajare and Vrushali A. Patil, Asian J. Pharm. Res. 2012; Vol. 2: Issue 3, Pg 111-117

[16] Rouge N, Leroux JC, Cole ET, Doelker E, Buri P. Prevention of the sticking tendency of floating minitablets filled into hard gelatin capsules. Eur J Pharm Biopharm. 1997;43:165-71.

[17] Sato Y, Kawashima Y, Takeuchi H, Yamamoto H. In vivo evaluation of riboflavin containing microballoons for floating controlled drug delivery system in healthy human volunteers. J Control Release. 2003;93:39-47. 